\title{
DETERMINAÇÃO DA CONCENTRAÇÃO MICELAR CRÍTICA DE ÁCIDOS HÚMICOS POR MEDIDAS DE CONDUTIVIDADE E ESPECTROSCOPIA
}

\author{
Solange Leite de Moraes* e Maria Olímpia Oliveira Rezende
}

Instituto de Química de São Carlos, Universidade de São Paulo, Av. Trabalhador Sãocarlense , 400, 13560-970 São Carlos - SP

Recebido em 22/7/03; aceito em 26/3/04; publicado na web em 27/7/04

\begin{abstract}
DETERMINATION OF THE CRITICAL MICELLE CONCENTRATION OF HUMIC ACIDS BY SPECTROSCOPY AND CONDUCTIMETRIC MEASUREMENTS. The aim of this study was the determination of the critical micelle concentration (CMC) of the sodium dodecyl sulfate (SDS) surfactant using spectroscopic and conductimetric determinations and to compare these methodologies in the determination of the CMC of different humic acids (HA). The CMC obtained by conductimetric determination was satisfactory. By spectroscopic determination two values of the CMC were obtained for HA. These values can be to due the intra and intermolecular interactions in the HA structure.
\end{abstract}

Keywords: spectroscopy and conductimetric determination; critical micelle concentration; humic acids.

\section{INTRODUÇÃo}

Tensoativos são moléculas anfifílicas caracterizadas por possuírem ambas as regiões estruturais hidrofílica e hidrofóbica, que dinamicamente se associam espontaneamente em solução aquosa a partir de uma determinada concentração denominada concentração micelar crítica $(\mathrm{CMC})^{1}$. Acima dessa concentração, as moléculas do tensoativo formam grandes agregados moleculares de dimensões coloidais. A esses agregados, que geralmente contem 60 a 100 moléculas do tensoativo, dá-se o nome de micelas ${ }^{2}$. Geralmente, em solução aquosa, as moléculas do tensoativo agregam-se formando uma esfera com caudas hidrofóbicas voltadas para o seu interior e os grupos hidrofílicos ou carregados, voltados para fora. Abaixo da CMC, o tensoativo está predominantemente na forma de monômeros ${ }^{2}$. A CMC depende da estrutura do tensoativo (tamanho da cadeia do hidrocarboneto) e das condições do meio (concentração iônica, contra-íons, temperatura etc. $)^{3-5}$. As micelas são termodinamicamente estáveis e facilmente reprodutíveis.

A formação de micelas é acompanhada por mudanças distintas em várias propriedades físicas tais como espalhamento de luz, viscosidade, condutividade elétrica, tensão superficial, pressão osmótica e capacidade de solubilização de solutos ${ }^{6-10}$.

Do ponto de vista analítico, uma das mais importantes propriedades dessas estruturas organizadas é a sua capacidade de solubilizar solutos de diferentes características. Esses solutos podem interagir eletrostaticamente, hidrofobicamente e pela combinação de ambos os efeitos ${ }^{11}$.

\section{Ácidos húmicos como tensoativos}

Um dos principais constituintes orgânicos presentes no solo são as substâncias húmicas. Essas substâncias consistem de uma mistura complexa de restos de animais e vegetais, em vários estágios de decomposição. No solo, essas substâncias possuem grande capacidade de retenção de calor, influenciando na germinação de raízes, além de possuírem papel importante no transporte de compostos orgânicos no ambiente. Devido a diferentes condições de formação, sua estrutura é indefinida podendo variar de região para região. As substânci-

*e-mail: sollm@ig.com.br as húmicas podem ser classificadas de acordo com a solubilidade em meio aquoso: ácido húmico, insolúvel em meio ácido e solúvel em meio alcalino; ácido fúlvico, solúvel em meio alcalino e em meio ácido e humina, insolúvel em ambos os meios ${ }^{12,13}$. Dentre essas frações, o ácido húmico (HA) está presente em maior quantidade.

Uma das mais importantes características dos HA é sua capacidade de solubilizar materiais hidrofóbicos ${ }^{12,14}$.

Os HA têm sido descritos na literatura com propriedades micelares, apresentando sítios hidrofílicos e hidrofóbicos que são responsáveis, por exemplo, pelo aumento da solubilidade de compostos orgânicos não polares em sistemas aquosos ${ }^{14,15}$. Essas estruturas de HA são responsáveis pelo fenômeno de agregação entre moléculas húmicas em função das condições do meio, como pH e concentração iônica, bem como interações com contaminantes orgânicos e inorgânicos ${ }^{15,16}$.

Wandruszca e Engebretson ${ }^{17}$ sugeriram que a característica tensoativa dos HA em água poderia ser atribuída à formação de pseudo micelas húmicas. Essas pseudo micelas húmicas são agregados de moléculas de HA que são análogas à formação de micelas por detergente. Esses compostos possuem núcleos não polares e superfícies polares. Sua estrutura é menos definida que em um detergente sintético, devido à variação da área e à composição do $\mathrm{HA}^{18}$; o efeito, contudo, é similar. Os HA como pseudo micelas podem formar ambos os processos: intra e intermolecular. No caso intramolecular, a forma do polímero espiral do HA dobra-se criando uma forma molecular que pode ser semelhante a um nó em uma corda ${ }^{19}$. O interior dessas estruturas contém sítios que retém poluentes orgânicos que são, deste modo, solubilizados e transportados ${ }^{20,21}$. O processo intermolecular envolve a associação de moléculas de HA, tanto grandes quanto pequenas. $\mathrm{O}$ efeito é novamente similar, contudo, requer grande concentração de HA. Ambos os processos de agregação são promovidos pela presença dos íons positivos na solução e pelo aumento na temperatura. Segundo Wandruszca e colaboradores ${ }^{22}$, o HA funciona melhor como tensoativo na presença de sais dissolvidos. Isso é comprovado nos casos em que o sal contém íons metálicos multivalentes como $\mathrm{Mg}^{2+}$. Íons monovalentes como $\mathrm{Na}^{+}$e $\mathrm{H}^{+}$também aumentam esse efeito, porém com menor intensidade ${ }^{16,22,23}$.

A estrutura do HA per si (considerando vários tipos de HA) também possui uma grande influência na propriedade tensoativa. A área molecular e a flexibilidade são importantes variáveis nessa conside- 
ração. HAs contendo estruturas grandes e mais flexíveis formam pseudo micelas mais eficientemente e são tensoativos melhores. Quando a estrutura do HA é reduzida em área, todas as formas de agregação de HA são menores. Moléculas pequenas não são adequadas para a formação de pseudo micelas, nesse caso, as características detergentes diminuem consideravelmente ${ }^{19,24}$.

A temperatura pode influenciar no comportamento do HA como tensoativo. Quando a temperatura da solução aumenta, os HAs tendem a se agregar mais, tornando-se tensoativos mais eficientes, até as proximidades da separação de fase. Esse fenômeno é denominado como "clouding", o qual é bem conhecido na química dos tensoativos ${ }^{25-28}$.

Tradicionalmente, os métodos envolvidos para a determinação da CMC são condutividade elétrica, tensão surperficial, espectrometria e fluorescência, dentre outros. Neste trabalho, utilizando-se a mesma metodologia desenvolvida para a determinação da CMC do dodecilsulfato de sódio (SDS), determinou-se a CMC do HA proveniente de turfa e de vermicomposto. As determinações desses valores foram feitas por medidas de condutividade e espectroscopia UVVIS.

\section{PARTE EXPERIMENTAL}

\section{Amostragem}

As amostras de HAs estudadas foram provenientes de turfa e vermicomposto. A turfa foi coletada a uma profundidade de 10 a 25 $\mathrm{cm}$, em uma turfeira às margens do rio Mogi-Guaçu, localizada nas proximidades do município de Luiz Antônio, no estado de São Paulo. O vermicomposto foi produzido a partir do esterco bovino tratado com Eisenia foetida. A extração do HA foi realizada segundo o procedimento da Sociedade Internacional de Substâncias Húmicas ${ }^{29}$ (adaptado).

\section{Determinação da CMC}

O tensoativo utilizado neste trabalho foi o $\operatorname{SDS}\left(\mathrm{C}_{12} \mathrm{H}_{25} \mathrm{OSO}_{3} \mathrm{Na}\right)$, de procedência Mallinckrodt.

As soluções de SDS e HA utilizadas para a determinação da CMC foram preparadas a partir de uma solução estoque de $50 \mathrm{mmol} /$ L e $200 \mathrm{mg} / \mathrm{L}$, respectivamente, em água. Para o preparo da solução de HA foi necessário adicionar uma pequena quantidade de $\mathrm{NaOH}$ $0,1 \mathrm{~mol} / \mathrm{L}$ para sua dissolução.

\section{Condutividade}

O condutivímetro utilizado foi o CD-21, Digimed. O equipamento foi calibrado com solução de $\mathrm{KCl} 0,01 \mathrm{~mol} / \mathrm{L}$. A condutância específica foi de $1411 \mu \mathrm{S} / \mathrm{cm}$, à temperatura de $25^{\circ} \mathrm{C}$.

Inicialmente adicionaram-se à cela condutimétrica cerca de 50 $\mathrm{mL}$ de água ultrapura, seguidos de alguns microlitos da solução estoque de SDS com o auxílio de uma micropipeta (Gilson, pipetman). Homogeneizou-se a mistura por meio de um agitador magnético. Para cada microlitro adicionado registrou-se uma medida. O mesmo procedimento foi realizado para a determinação da CMC do HA em solução aquosa.

\section{Espectroscopia UV-VIS}

O método de determinação da CMC por espectroscopia de absorção UV-VIS baseia-se na variação do espectro de absorção do corante em função da concentração do tensoativo adicionado para uma concentração fixa do corante.
A partir da solução estoque de SDS $50 \mathrm{mmol} / \mathrm{L}$ em água obtiveram-se as seguintes concentrações: $2 ; 3 ; 4 ; 5 ; 6 ; 8 ; 10 ; 15 ; 20 ; 25$ e $30 \mathrm{mmol} / \mathrm{L}$. Os sistemas de diluições utilizados foram água ultrapura, $10 \mathrm{mmol} / \mathrm{L}$ de tampão fosfato de sódio $\mathrm{pH}$ 7,0 e $5 \mathrm{mmol} / \mathrm{L}$ de tampão borato de sódio $\mathrm{pH} 9,3$.

Para os estudos com HA partiu-se da solução estoque de HA $200 \mathrm{mg} / \mathrm{L}$ em água (a que foi adicionada uma pequena quantidade de $\mathrm{NaOH} 0,1 \mathrm{~mol} / \mathrm{L}$ para dissolver o HA) e as seguintes concentrações foram obtidas: $8 ; 16 ; 20 ; 22 ; 24 ; 28 ; 32 ; 36 ; 40 ; 44 ; 48 ; 52 ; 56$ e $60 \mathrm{mg} / \mathrm{L}$. Os mesmos sistemas de diluição utilizados para o SDS foram utilizados para o HA.

Os valores de concentrações de SDS e HA foram escolhidos acima e abaixo do valor teórico da CMC no sistema aquoso, que para o SDS é $8 \mathrm{mmol} / \mathrm{L}^{30}$ e para o HA é aproximadamente $30 \mathrm{mg} / \mathrm{L}^{19}$, dependendo da sua natureza.

Os espectros na região do visível foram obtidos com um espectrofotômetro Hitachi, modelo U3501. O corante sudan III (504 $\mathrm{nm}$ ) foi utilizados para a identificação das micelas.

\section{RESULTADOS E DISCUSSÃO}

\section{Determinação da CMC por condutividade}

Inicialmente foi determinada a CMC do SDS a fim de se estabelecer um método de análise utilizando um tensoativo que já possuía um valor teórico determinado e, a seguir, expandir a metodologia para a determinação da CMC do HA.

$\mathrm{O}$ valor da CMC do SDS determinado por condutividade foi obtido pela intercessão de duas retas. A Figura 1 mostra a variação da condutância em função da concentração de SDS adicionado em água.

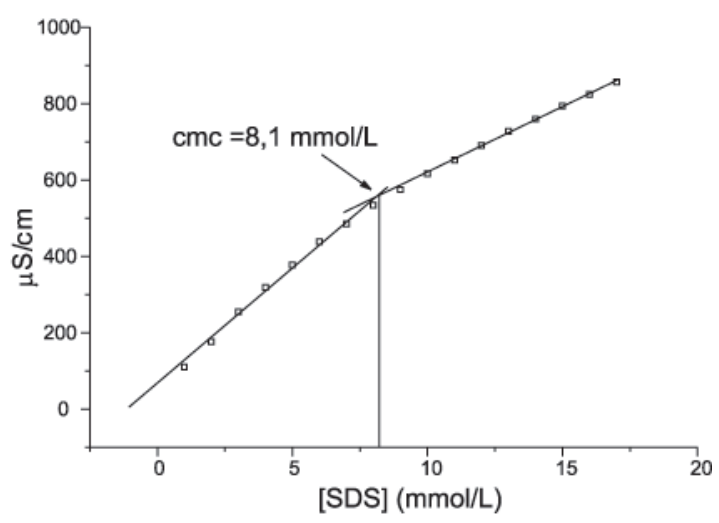

Figura 1. Variação da condutância em função da concentração de SDS adicionado em água à $25^{\circ} \mathrm{C}$

As duas retas correspondem ao estado de monômero e micela do tensoativo. O ponto de intercepção corresponde ao valor da CMC. Antes da CMC, a variação da condutividade é devida à adição de moléculas do tensoativo à solução. Após a CMC, a variação da condutividade deve-se ao aumento de micelas no meio e a partir da CMC a concentração do monômero permanece constante.

O valor da CMC do SDS em água através de medidas de condutividade foi $8,1 \mathrm{mmol} / \mathrm{L}$, concordante com o valor teórico ${ }^{30}$.

A Figura 2 apresenta a variação da condutância em função da concentração do HA de turfa e de vermicomposto, em água. Os resultados obtidos para a CMC através da intercessão das retas para o HA de turfa e de vemicomposto em solução aquosa ( $\mathrm{pH} 6,8)$ foram 36,0 e $28,0 \mathrm{mg} / \mathrm{L}$, respectivamente. À medida que se aumentou a concentração de HA, houve um aumento nas medidas de condutividade, similar ao que ocorreu com o SDS. 

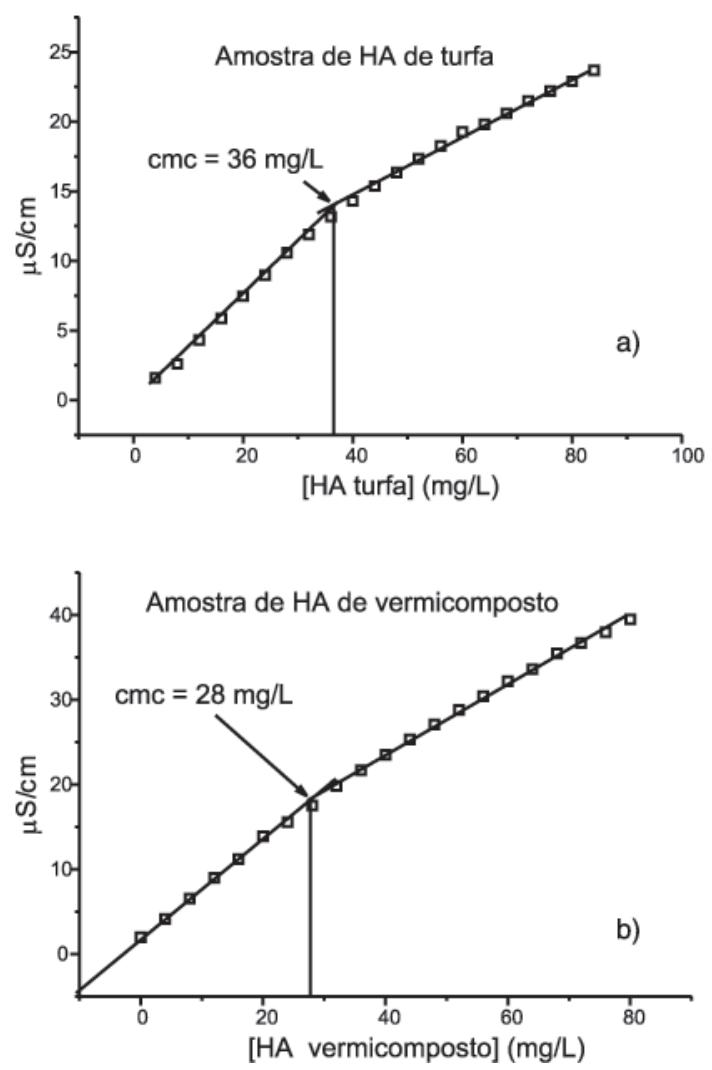

Figura 2. Variação da condutância em função da concentração do HA de turfa (a) e HA de vermicomposto (b) adicionados em água

\section{Determinação da CMC por espectroscopia de absorção UV-VIS}

Os valores da CMC por espectroscopia de absorção UV-VIS foram determinados por mudanças de comportamento na curva de absorção. Essa mudança de comportamento ocorre, por sua vez, devido a mudanças no ambiente em que essas moléculas se encontram. Aumentando-se a concentração de SDS, há formação de micelas e, com isso, ocorre a incorporação do corante na micela, até um ponto onde o corante está totalmente solubilizado na micela, obtendo-se, assim, o ponto máximo da curva. O ponto máximo representa o valor da CMC. A Figura 3 apresenta as medidas de absorção para diferentes concentrações de SDS nos diferentes sistemas estudados.

Os valores obtidos da CMC do SDS para os diferentes sistemas estudados (água, tampão fosfato de sódio e tampão borato de sódio) foram 6,0, 5,0 e 5,0 $\mathrm{mmol} / \mathrm{L}$, respectivamente.

Observou-se que o valor da CMC diminui na presença da solução tampão. Isso ocorre devido à presença de sal, uma vez que os íons do sal neutralizam as cargas na superfície da micela, diminuindo a atmosfera iônica ao redor da cabeça iônica do tensoativo e a repulsão eletrostática entre eles.

A determinação da CMC para o HA foi feita seguindo o mesmo procedimento para o SDS, variando-se a concentração de HA em diferentes sistemas de tampão e medindo-se a absorbância. Contudo, o comportamento do HA não foi similar ao do SDS (Figura 4), visto que não houve apenas uma mudança brusca na curva, o que indicaria o valor da CMC e a formação das micelas. O que se observou foram duas mudanças na curva de absorção o que, neste caso, corresponderia a dois prováveis valores de CMC para o HA. Este mesmo comportamento foi observado para a amostra de HA de vermicomposto, Figura 5. Nota-se que os valores da CMC obtidos dependem da natureza do HA.
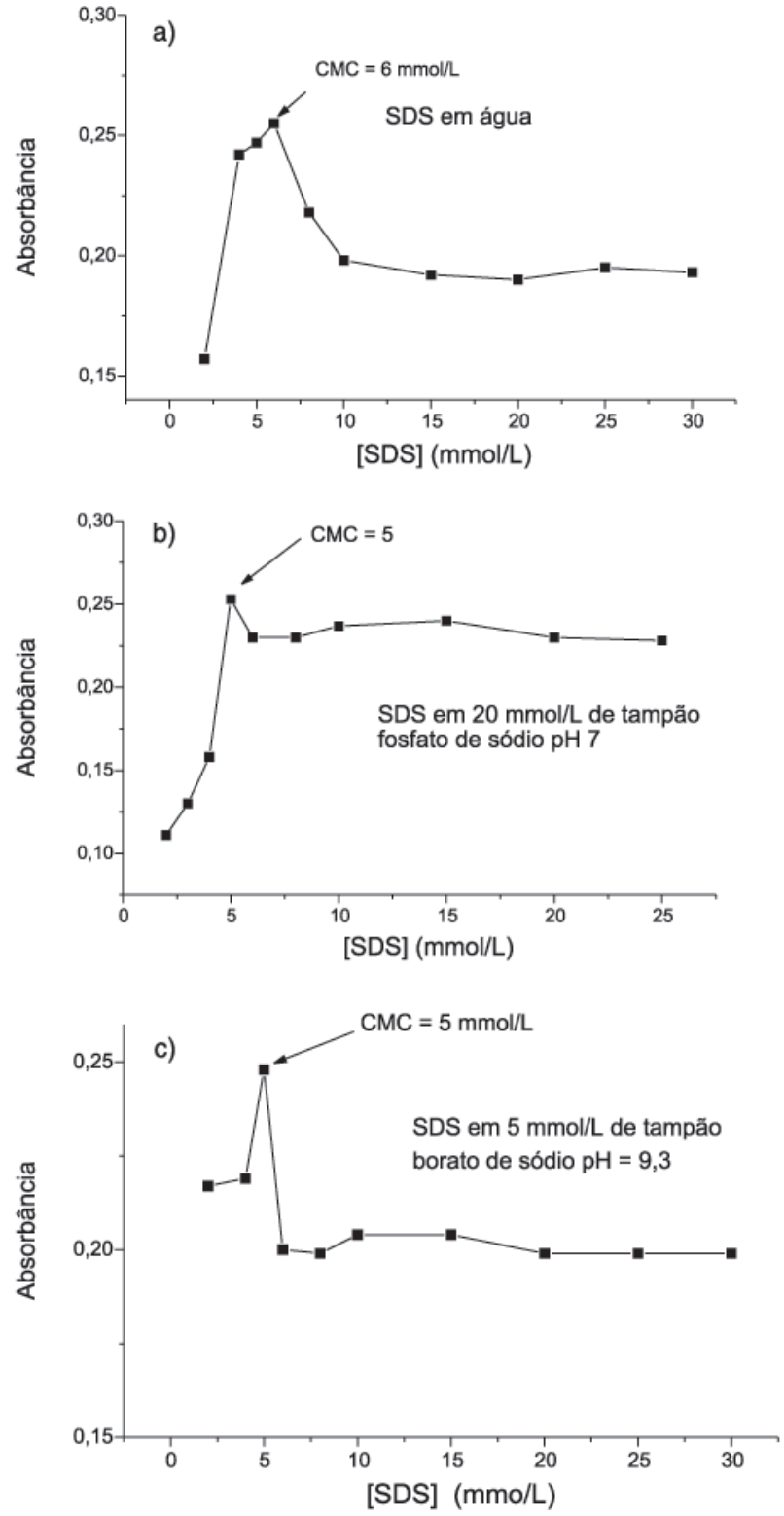

Figura 3. Medidas de absorbância para diferentes concentrações de SDS utilizando o corante sudan III (504nm) em diferentes solventes: (a) água e (b) $10 \mathrm{mmol} / \mathrm{L}$ de fosfato de sódio $\mathrm{pH} 7$ e (c) $5 \mathrm{mmol} / \mathrm{L}$ de borato de sódio $\mathrm{pH} \mathrm{9,3}$

Pelas determinações espectroscopicas obtiveram-se 2 valores para a CMC para ambas as amostras de HA, já que houve duas mudanças na curva de absorção. Visto que as moléculas de HA são polidispersivas e que possuem agregação intra e intermolecular, os dois valores da CMC encontrados por espectroscopia podem corresponder às agregações intra e intermolecular, resultando em duas regiões específicas nas quais pode ocorrer a formação de estruturas pseudo micelares. O menor valor da CMC sugere interações intramoleculares enquanto que o maior valor estaria associado às interações intermoleculares, uma vez que esse processo requer maior concentração de HA. Os valores obtidos por espectroscopia foram independentes do sistemas de tampão usado, mostrando que não houve influência direta do tampão com os valores de CMC.

Os dois valores obtidos para a CMC por espectroscopia são devidos à incorporação do corante ao agregado micelar, tornando-se possível a identificação da pseudo micela húmica pelo processo intramolecular. 

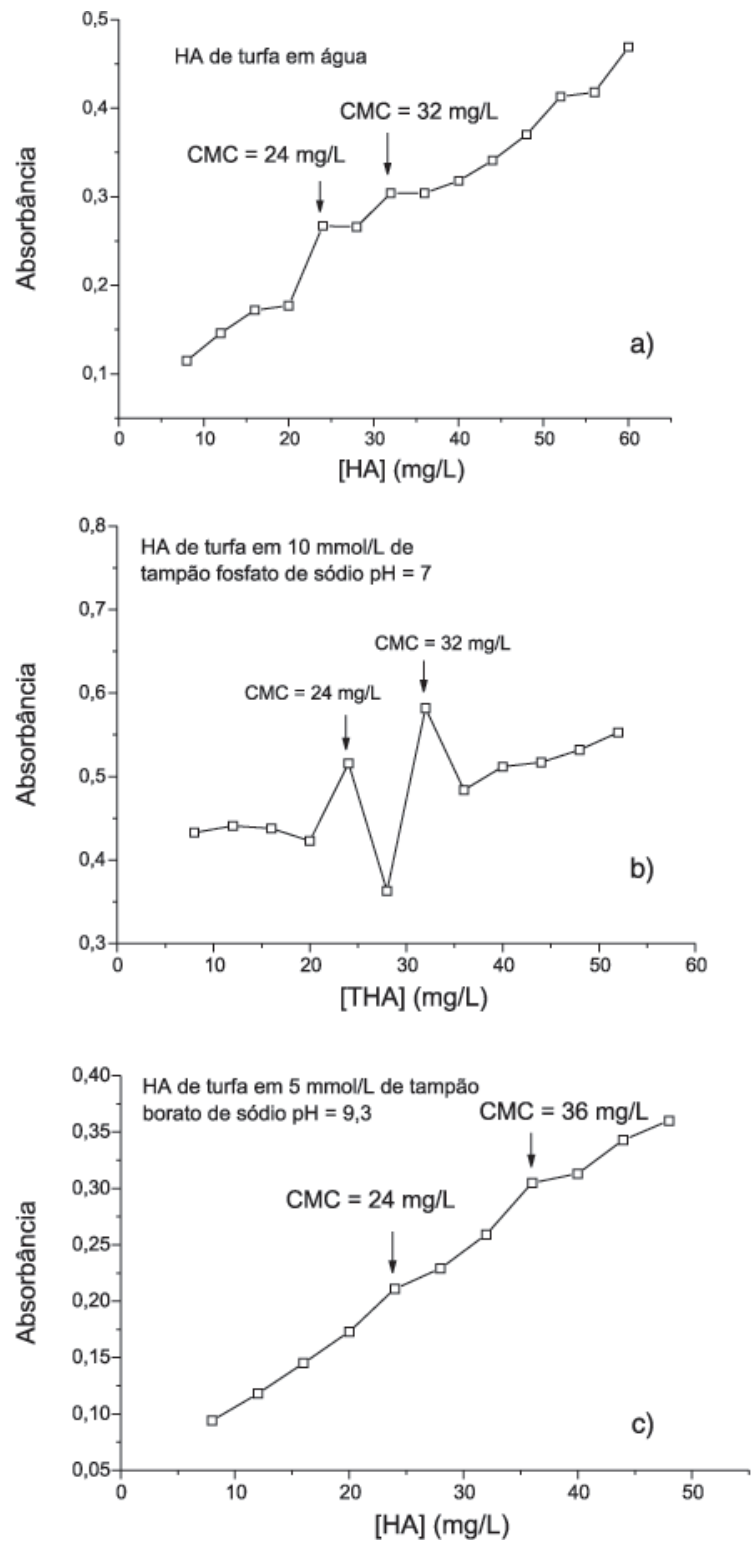

Figura 4. Medidas de absorbância para diferentes concentrações de HA de turfa utilizando o corante sudan III $(504 \mathrm{~nm})$ em diferentes solventes: (a) água, (b) $10 \mathrm{mmol} / \mathrm{L}$ de tampão fosfato de sódio $\mathrm{pH} \mathrm{7,0,} \mathrm{(c)} 5 \mathrm{mmol} / \mathrm{L}$ de borato de sódio $\mathrm{pH} 9,3$

\section{CONCLUSÕES}

A determinação da CMC para o HA foi dependente da estrutura do HA e do meio em que ele se encontrava. Considerando-se as duas metodologias testadas para a determinação da CMC para o HA, verificou-se que pelas medidas de condutividade, o HA teve um comportamento similar ao do SDS, sendo possível a determinação da sua CMC em água. No entanto, considerando as medidas espectroscópicas foram encontrados dois possíveis valores para a CMC, devido ao HA ser uma molécula muito complexa, propiciando agregações inter e intramoleculares do corante com o agregado micelar.

\section{AGRADECIMENTOS}

As autoras agradecem à FAPESP e ao CNPq pelo apoio financeiro. Solange L. de Moraes agradece à FAPESP (processo \# 00/ 02368-5) pela bolsa de doutorado concedida.
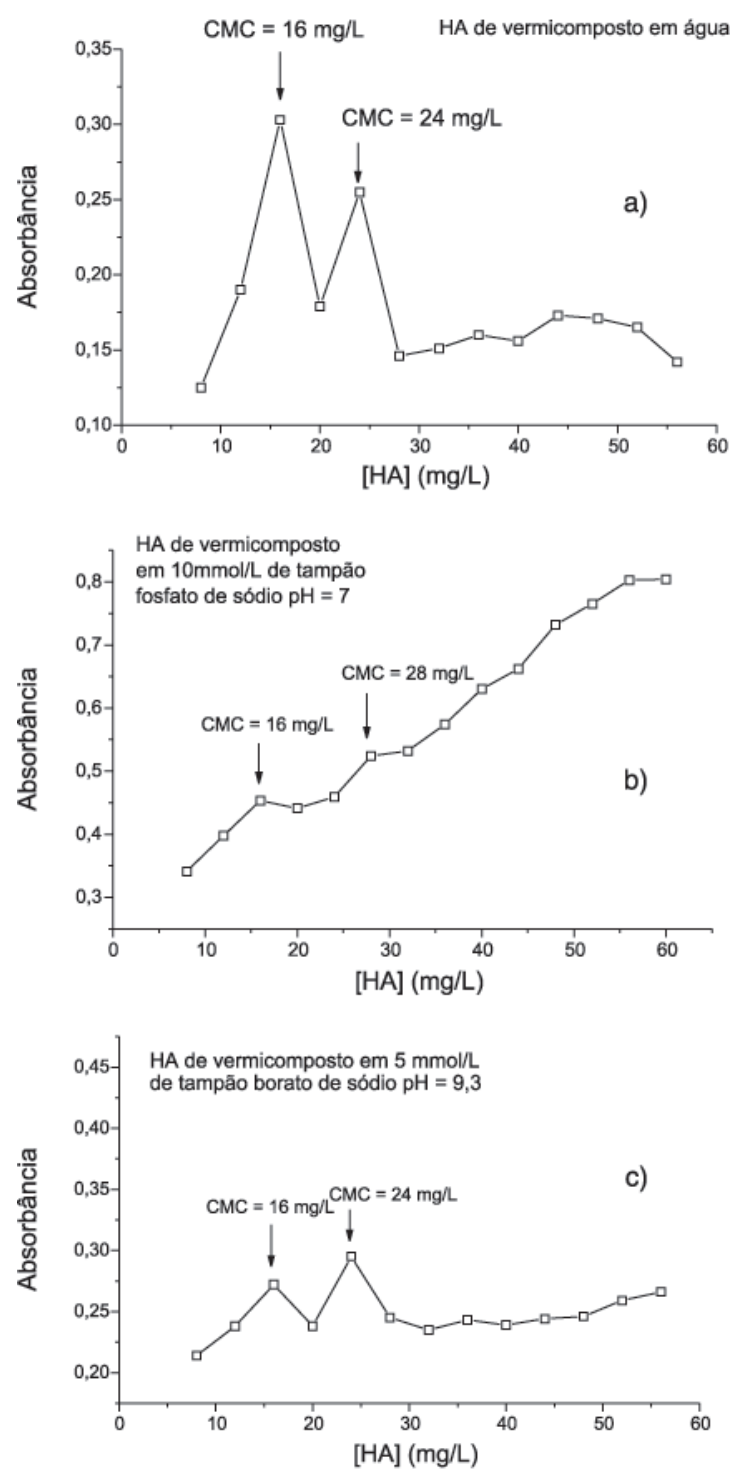

Figura 5. Medidas de absorbância para diferentes concentrações de HA de vermicomposto utilizando o sudan III $(504 \mathrm{~nm})$ em diferentes solventes: (a) água, (b) $10 \mathrm{mmol} / \mathrm{L}$ de tampão fosfato de sódio $\mathrm{pH} \mathrm{7,} \mathrm{(c)} 5 \mathrm{mmol} / \mathrm{L}$ de borato de sódio $\mathrm{pH}$ 9,3

\section{REFERÊNCIAS}

1. Martinéz, R. C.; Gonzalo, E. R.; Cordero, B. M.; Pavón, J. L.; Pinto, C. G.; Lespada, E. F.; J. Cromatogr., A 2000, 902, 251.

2. Maniasso, N.; Quim. Nova 2001, 24, 87.

3. Jacquier, J. C.; Desbène, P. L.; J. Cromatogr., A 1995, 718, 167.

4. Lin, C. E.; Wang, T. Z.; Chiu, T.C.; Hsuch, C. C.; J. Resol. Chromatogr. 1999, 22, 265 .

5. Tesarová, E.; Tuzar, Z.; Nésmerák, K.; Bosáková, Z.; Gás, B.; Talanta 2001, $54,643$.

6. Akhter, M. S.; Colloids Surf., A 1997, 121, 103.

7. Benito, I; García, M. A.; Monge, C.; Saz, J. M.; Marina, M. L.; Colloids Surf., A 1997, 125, 221.

8. Ysambertt, F.; Vejar, F.; Parede, J.; Salager, J. L.; Colloids Surf., A 1998, 137, 189.

9. Cimentes, A.; Bemal, J. L.; Diez-Masa, J. C.; Anal. Chem. 1997, 69, 4271.

10. Ma, G.; Li, G.; Xu, Y.; Wang, H.; Ye, X.; Colloids Surf., A 1998, 143, 89.

11. Mrestani, Y.; Neubert, R.; Rüttinger, H. H.; J. Cromatogr., A 2000, 868 , 317.

12. Eauen, T. G.; Debacher, N .A.; Sierra, M. M.; Sierra, E. J. S.; Quim. Nova 2002, 25, 909 . 
13. Piccolo, A.; Soil Sci. 2001 166, 810.

14. Hayes, M. H. B.; Clapp, C. E.; Soil Sci. 2001, 166, 723.

15. Wershaw, R.; Environ. Sci. Technol. 1998, 27, 14.

16. Fetsch, D.; Hradilová, M.; Méndez, E. M. P.; Havel, J.; J. Chromatogr., A 1998, 817,313

17. Wandruszka, R. V. ; Engebretson, R.; Talanta 1997, 44, 805.

18. Yates, L. M.; Wandruszka, R. V.; J. Soil Sci. Soc. Am. 1999, 63, 1645.

19. Schmitt, Ph.; Freitag, D.; Garrison, A. W.; Schiavon, N.; Kettrup, A.; Chemosphere 1997, 35, 55.

20. Piccolo, A.; Nardi, S.; Concheri, G.; Chemosphere 1996, 33, 595.

21. Ferreira, J. A.; Nascimento, O. R.; Martin, L.; Environ. Sci. Technol. 2001, $35,761$.

22. Morra, M. L.; Corapcioglu, M. O.; Wandruszka, R. V.; Marshall, D. B.; Topper, K.; J. Soil Sci. Soc. Am. 1990, 54, 1283.
23. Kopinke, F. D.; Georgi, A.; Mackenzie, K.; Anal. Chim. Acta 1997, 31, 101.

24. Engebretson, R. R.; Wandruszka, R. V.; Environ. Sci. Technol. 1998, 32, 488.

25. Engebretson, R. R.; Wandruszka, R. V.; Environ. Sci. Technol. 1994, 28, 1934.

26. Engebretson, R. R.; Amos, T.; Wandruszka, R.V.; Environ. Sci. Technol. 1996, 30,990

27. Toerne, K.; Rogers, R.; Wandruszka, R. V.; Langmuir 2001, 17, 6119.

28. Engebretson, R. R.; Wandruszka, R. V.; Environ. Sci. Technol. 1999, 33, 4299.

29. Schnitzer, M. Em Soil Organic Matter; Schnitzer, M.; Khan, S. U., eds., Elsevier: Amsterdam, 1978, p. 1-64.

30. Weinberg, R.; Pratical capillary electrophoresis, Academic Press, In.: New York, 1993, p. 305 